Analytical Methods

\title{
Detection of coffee flavour ageing by solid-phase microextraction/surface acoustic wave sensor array technique (SPME/SAW)
}

\author{
Nicole Barié ${ }^{\mathrm{a}}$, Mark Bücking ${ }^{\mathrm{b}}$, Ullrich Stahl ${ }^{\mathrm{c}}$, Michael Rapp ${ }^{\mathrm{a}, *}$ \\ ${ }^{a}$ Karlsruhe Institute of Technology (KIT), Institute for Microstructure Technology (IMT), Hermann-von-Helmholtz-Platz 1, 76344 Eggenstein-Leopoldshafen, Germany \\ ${ }^{\mathrm{b}}$ Fraunhofer Institute for Molecular Biology and Applied Ecology (IME), 57392 Schmallenberg, Germany \\ ${ }^{\mathrm{c}}$ Facultad de Ingeniería Química, Universidad Central del Ecuador, Quito, Ecuador
}

\section{A R T I C L E I N F O}

\section{Article history:}

Received 17 July 2013

Received in revised form 3 September 2014

Accepted 11 December 2014

Available online 17 December 2014

\section{Keywords:}

Coffee ageing

Flavour

Sensors

SAW

\begin{abstract}
A B S T R A C T
The use of polymer coated surface acoustic wave (SAW) sensor arrays is a very promising technique for highly sensitive and selective detection of volatile organic compounds (VOCs). We present new developments to achieve a low cost sensor setup with a sampling method enabling the highly reproducible detection of volatiles even in the ppb range. Since the VOCs of coffee are well known by gas chromatography (GC) research studies, the new sensor array was tested for an easy assessable objective: coffee ageing during storage. As reference method these changes were traced with a standard GC/FID set-up, accompanied by sensory panellists. The evaluation of GC data showed a non-linear characteristic for single compound concentrations as well as for total peak area values, disabling prediction of the coffee age. In contrast, the new SAW sensor array demonstrates a linear dependency, i.e. being capable to show a dependency between volatile concentration and storage time.
\end{abstract}

(c) 2014 Elsevier Ltd. All rights reserved.

\section{Introduction}

In routine analysis wet chemical methods are often used for the determination of food quality. In addition, instrumental methods and sensory analysis are described as usable tools for the determination of the storage period, the quality and the origin. Unfortunately, apart from the time-consuming aspect of these methods, they are sensitive to misinterpretation. In the last decade new devices, so called "electronic noses", have been described in the literature as a both cost-effective and timesaving substitute for these methods. Most of these chemical sensors showed deficiencies in selectivity, sensitivity and reproducibility and only a few systems are classified to partly replace the classical methods (Bücking, Haugen, \& Steinhart, 2003).

The aim of the present investigation was to study the analytical capability of a new system which combines Solid Phase Micro Extraction (SPME) with a newly developed Surface Acoustic Wave (SAW) based sensor system. The combination of a headspace autosampler with sample injection via SPME fibre is frequently used as a sampling method in classical gas chromatography (GC). The latter of course involves a proper detector unit at the column

\footnotetext{
* Corresponding author. Tel.: +49 7216082 3238; fax: +49 72160826667.

E-mail address: michael.rapp@kit.edu (M. Rapp).
}

output as well. Instead of using an expensive and a relatively slow GC plus attached detector, this study uses a self-developed miniaturised sensor array based on eight differently coated SAW devices as detection unit. Since such sensors provide some semi-selectivity, the necessity of a laborious and time intensive GC separation can be omitted. SAW devices have already proved to be suitable in many technical applications, above all in electronics, electronic data processing, and high-frequency technology. They have the advantages of being low-cost, simple in handling and are small sized. Typically, they are operated at an ultrasonic range of frequencies from $10 \mathrm{MHz}$ to $2 \mathrm{GHz}$. Sensors on the basis of various different acoustic wave types, which differ in terms of boundary conditions and the direction of their deflection components, have been already implemented for detection purposes in gaseous as well as in liquid media, e.g. quartz crystal microbalances (Nakanishi, Muguruma, \& Karube, 1996), sensors based on Rayleigh waves (Wohltjen \& Dessy, 1979), acoustic plate modes (Martin, Ricco, Niemczyk, \& Frye, 1989), transverse surface waves (Baer, Flory, Tom-Moy, \& Solomon, 1992), and Love waves (Kovacs, Vellekoop, Haueis, Lubking, \& Venema, 1994). For sensing, such devices were typically equipped with selective coatings which interact with the ambient medium by physical absorption. Installed into an oscillator circuit the sensors serve as the frequency determining element. The interaction of the acoustic 
wave with the surrounding medium then causes the velocity and amplitude of the wave to change, which is indicated by a change in the resonance frequency of the oscillator. Thus, changes in the mass density, mechanical stiffness or viscosity can be monitored as a sensing response. However, the major measurement parameter generally is the change in resonance frequency due to the change in mass, which results from the interaction between analyte and sensitive coating. To achieve selectivity towards a certain analyte, several sensor devices must then be combined properly forming a sensor array. An array of polymer coated SAW sensors with each sensor having a different sensitive coating, combined with a suitable data processing, allows retrieving the qualitative as well as the quantitative composition of the volatiles of interest. Therefore, it is a very promising technique for highly sensitive and selective organic gas detection and electronic nose applications. New developments in the fabrication of SAW sensors, e.g. coating and sampling techniques, enable a highly reproducible detection of volatiles in the ppm and ppb range.

In the past we already presented a novel concept to achieve a miniaturized SAW sensor array comprising a dramatic reduction of sampling volume and inner surface area which minimises response time and unwanted memory effects (Bender, Barié, Romoudis, Voigt, \& Rapp, 2003; Rapp, Reibel, Voigt, Balzer, \& Bülow, 2000). For an on-line control of VOC's this system showed already an excellent long-term stability in combination with good reproducibility and selectivity (Wessa, Küppers, Rapp, \& Reibel, 2000).

SPME as a sample preparation technique for extraction of organic compounds from complex samples was introduced by Arthur and Pawliszyn in 1990 (Pawliszyn, 1997) and further developed in 1993 as headspace-SPME (Pawliszyn, 1999). This method can concentrate volatile and non-volatile compounds from liquid, gas, and solid matrices for chromatographic analyses. A SPME unit comprises a narrow diameter fused silica fibre coated with a thin sorbent phase; usually a polymeric material (e.g. polydimethylsiloxane (PDMS), polyacrylate, and divinyl benzene), in some cases mixed with a solid adsorbent (e.g. porous carbon). The fibre is attached to a stainless steel plunger, which moves the coated fibre within a protective needle. For extraction the fibre is exposed to the sample or its headspace, from which the analytes are concentrated directly by adsorption on the sorbent phase. The method integrates sampling, extraction, concentration, and sample introduction into the chromatography system in a single step and can be automated routinely. It provides lower detection limits than other techniques, and dramatically reduces sample preparation time. The SPME technique has been described and validated for numerous applications in several areas, including chemical, environmental, pharmaceutical, aroma, food, and natural products analysis (Pawliszyn, 1997, 1999; Pelusio et al., 1995; Snow \& Slack, 2002; Yang \& Peppard, 1995). Coupling the automated SPME technique with a SAW based microarray should lead to a powerful tool for a wide range of applications.

Because of its high commercial impact in the food industry, coffee was selected as food material for this study. State of the art on coffee analysis is the use of SPME in conjunction with GC and mass spectrometry (MS) as detector unit (López-Galilea, Fournier, Cid, \& Guichard, 2006; Mondello et al., 2005). With this method even single components can be determined as well classified as complex composition in conjunction with added statistical analysis ( $M$. Korhonová, D. Klimcíková, P. Bednár, \& Coffee aroma-statistical analysis of compositional data, 2009). Such methods necessarily require highly expensive equipment and a well trained staff. In order to simplify the process of analysis, coffee volatiles can also be analysed with gas chromatographic techniques using standard detectors (Bücking \& Steinhart, 2002; Semmelroch \& Grosch, 1996). Some publications introduced already alternative detector techniques in conjunction with the use of the SPME technique in coffee research (Bicchi, Panero, Pellegrino, \& Vanni, 1997; Costa Freitas, Parreira, \& Vilas-Boas, 2001). Furthermore, coffee aroma has been always a topic for the so called "electronic nose" devices which correspond to a semi-selective detector system completely omitting GC as separation technique (Gretsch et al., 1997; Pardo et al., 2000). Most of the publications in this field only concentrate onto the differentiation between the two major varieties of coffee plants, Arabica and Robusta. The interest in our new study concentrates towards a more complex question: the coffee ageing. Coffee freshness is mainly caused by certain low-boiling components, e.g. sulphur compounds, Strecker aldehydes and di-ketones. During storage, coffee is losing its typical and pleasant flavour, and therefore its attraction for the consumer. In this paper we test and compare the reliability of data obtained by means of 3 completely different methods:

1. New sensor system as detecting device in conjunction with the SPME technique.

2. Conventional method using a trained human panel using the human nose by sniffing as the classic way of aroma judgement.

3. Standard laboratory method using a commercial GC instrumentation with MS or FID detector.

\section{Materials and methods}

\subsection{Materials}

Coffee: a commercially available coffee blend, purchased from a local supermarket, was analysed. The coffee beans were ground in a household coffee grinder and $10 \mathrm{~g}$ of the sample were stored in $50 \mathrm{ml}$ headspace vials for 2-14 days. In order to achieve a realistic household-like storage condition these vials were only capped but not sealed, i.e. a slight exchange of air was permanently possible.

Chemicals: compounds were obtained from Aldrich (Steinheim, Germany) and ACROS (Gelnhausen, Germany).

\subsection{Instrumentation}

\subsubsection{Gas chromatography}

GC/MS: $2 \mathrm{~g}$ of coffee powder were filled in an external dynamic Headspace device, nitrogen was flushed at $40 \mathrm{ml} / \mathrm{min}$ for $5 \mathrm{~min}$ and the volatiles were collected on a Tenax TA tube. Volatiles were desorbed from the Tenax TA tubes by the use of a thermal desorber system TDS 2 (Gerstel, Mühlheim a. d. R., Germany) and injected into a CIS 3 cold injection system (Gerstel) with nitrogen cooling $\left(-150^{\circ} \mathrm{C}\right)$. To start the GC run, the trap was heated up very rapidly to $300^{\circ} \mathrm{C}$. GC/MS analysis was performed with a GC 8000 system (Interscience), coupled with an Automass II mass spectrometer (ThermoFinnigan, Breda, The Netherlands) run in the electron impact mode at $70 \mathrm{eV}$. A CP-Sil8 column, Varian, Bergen op Zoom, The Netherlands (100\% dimethylpolysiloxane, $30 \mathrm{~m} \times 0.25 \mathrm{~mm}$ i.d., $0.5 \mu \mathrm{m}$ film thickness) and the following temperature program was used: $37^{\circ} \mathrm{C}$ held for $7 \mathrm{~min}$, raised at $4^{\circ} \mathrm{C} / \mathrm{min}$ to $200^{\circ} \mathrm{C}$, then raised at $8{ }^{\circ} \mathrm{C} / \mathrm{min}$ to $250^{\circ} \mathrm{C}$, and held for $2 \mathrm{~min}$. Helium was used as carrier gas with a constant flow of $1.0 \mathrm{ml} / \mathrm{min}$. The identification of the compounds was achieved by comparison of retention data on $\mathrm{CP}-\mathrm{Sil} 5$ and CP-Sil8 columns and mass spectrometric data, as well as comparison with the authentic reference substances.

GC/FID: $2 \mathrm{~g}$ of coffee powder were filled in a $20 \mathrm{ml} \mathrm{HS}$-vial. After an incubation time of $15 \mathrm{~min}$ at $30^{\circ} \mathrm{C}$ and/or $60^{\circ} \mathrm{C}$ in the incubator unit of a CombiPal autosampler (CTC, Switzerland), $2.5 \mathrm{ml}$ of Headspace volume were injected into a Trace GC with a PTV Injector system and FID (Interscience, Breda, The Netherlands). A CP-Sil 5 column, Varian (100\% dimethylpolysiloxane, $100 \mathrm{~m} \times 0.53 \mathrm{~mm}$ i.d., $5 \mu \mathrm{m}$ film thickness) and the following temperature program was used: $30^{\circ} \mathrm{C}$ held for $7 \mathrm{~min}$, raised at $4{ }^{\circ} \mathrm{C} / \mathrm{min}$ to $200^{\circ} \mathrm{C}$, then 
raised at $8^{\circ} \mathrm{C} / \mathrm{min}$ to $250{ }^{\circ} \mathrm{C}$, and held for $2 \mathrm{~min}$. Helium was used as carrier gas with a constant flow of $8.8 \mathrm{ml} / \mathrm{min}$.

\subsubsection{Sensory experiments}

A panel of six trained assessors performed a "Triangular" test according to an official German standard protocol (Deutsches Institut für Normung e.V, 1995) with samples of the stored coffee powder in random. The assessors had to find the sample that was different by sniffing.

\subsubsection{Sensor system}

The devices used for this work are based on a Surface Transverse Wave (STW) resonator design (I.D. Avramov, A. Voigt, M. Dirschka. Comparative studies on polymer coated SAW, Kansas City, \& USA, 2000). The devices work at an operation frequency of $433 \mathrm{MHz}$. Since this frequency is relatively high compared to that of quartz microbalances (QMB) or even other surface acoustic wave based chemical sensors (Grate, Martin, \& White, 1993a, 1993b), a high sensitivity can be expected. On the other hand, if that high sensitivity is not really required, e.g. in case of an enriched headspace analysis, it allows in turn the use of very thin coatings providing faster response times. The STW sensors used were coated with commercially available polymers. A wide variety of polymer materials was tested. Finally, a convenient set of coating materials was found consisting of polymers like polyisobutylene (PIB), polyepichlorohydrine (PECH), and poly(butyl methacrylate) (PBMA) which are also often included in other comparable sensor arrays (Wohltjen, 1984). These 3 materials were supplemented with further 5 materials to complete the set of 8 sensors involved in the used sensor array. These additional materials were also commercially available but were complex mixtures usually used for other purposes: 2 different adhesives and 2 different commercial paints provided from simple aerosol cans. All 8 materials were solved in toluene and then spray-coated onto the SAW substrate surface until a frequency shift of about $1 \mathrm{MHz}$ was observed due to the precipitated polymer film in the dry state (toluene free), respectively. With such a full set of semi-selective coating materials a high chemical selectivity can be achieved if pattern recognition methods are used for proper array data evaluation.

The reason for the use of special STW-type SAW devices is their ability to permit high coating thicknesses, thus providing a higher sensitivity. Additionally, these devices may support the so called Love wave effect (Barié, Wessa, Bruns, \& Rapp, 2004): for a certain thickness value in the range of a few $\mu \mathrm{m}$ the coating may also act as a resonating waveguide structure. In this case a related STW device would become a very sensitive Love wave device (Barié et al., 2004). However, in the special case of coffee aroma detection it may not be wise to increase the coating thickness to a large extend since, on the other hand, this means also a significant increase in sorption time. The ability of the system to detect small concentrations of analytes depends on the sensitivities of the individual devices, on a proper sampling strategy (see below) and on the accuracy of the applied pattern recognition.

Micro array: eight STW devices were combined into a self developed miniaturized sensor array, each device being coated with a different polymer layer. The frequencies of the devices were read out consecutively using a multiplexing technique (Bender, Länge, Voigt, \& Rapp, 2004). A ninth, uncoated device was used as a reference, thus only the differential frequencies must be processed rather than the high frequencies of operation. The read out speed of the eight frequency values was $1 \mathrm{~s}$ leading to a refresh rate frequency of $1 \mathrm{~Hz}$. The heart of the system is a miniaturized sensor array on a small high frequency circuit board with incorporated (milled) and gold plated gas channels. The latter contains a total gas volume of only $80 \mu \mathrm{l}$. The sensor devices are inserted facedown onto the gas channels, their sensing surfaces being in contact with the gas flow. The devices are contacted capacitively to the circuit board. This way neither bonding wires nor absorbing materials like glue are needed to keep the sensors contacted and in place. An

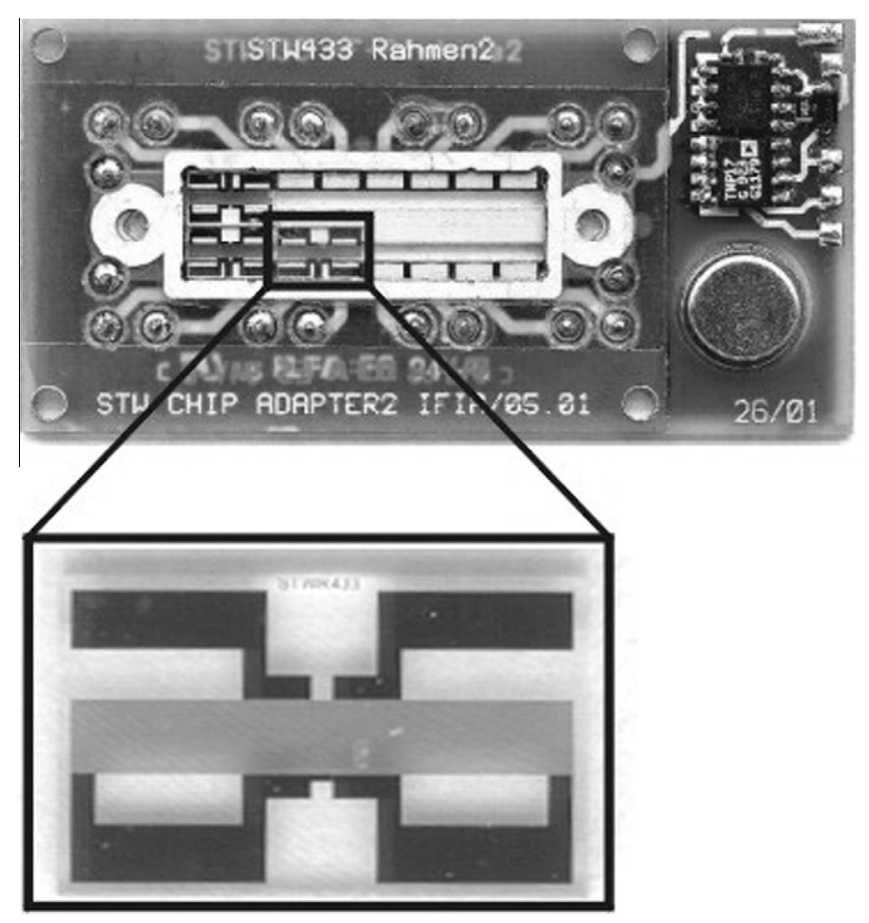

Fig. 1. Photograph of the SAW micro array with three sensor devices inserted (top view, cover removed). The dimensions of the board are $6 \mathrm{~cm} \times 3 \mathrm{~cm}$. Below: photograph of the STW433 device.

Table 1

Volatile compounds identified in headspace gas of ground coffee.

\begin{tabular}{|c|c|c|}
\hline Component & $\mathrm{RT}^{\mathrm{c}}$ & $\mathrm{KI}^{\mathrm{C}}$ \\
\hline 2,3-Butandione $\mathrm{a}^{\mathrm{a}}$ & 4.84 & 602 \\
\hline Acetic acid & 5.71 & 613 \\
\hline 3-Methylbutanal ${ }^{\mathrm{a}}$ & 7.85 & 652 \\
\hline 2-Methylbutanal ${ }^{\mathrm{a}}$ & 8.40 & 662 \\
\hline Hydroxy-2-propanone & 8.61 & 666 \\
\hline 2,3-Pentandione $\mathrm{a}^{\mathrm{a}}$ & 10.18 & 695 \\
\hline 2,5-Dimethylfuran & 10.94 & 708 \\
\hline 3-Hydroxy-2-butanone & 11.10 & 711 \\
\hline Phenol & 11.88 & 724 \\
\hline Pyrazine & 12.21 & 730 \\
\hline Pyridine & 13.09 & 746 \\
\hline Pyrrole & 13.66 & 756 \\
\hline 2,3-Hexadione & 15.27 & 784 \\
\hline Hexanal & 16.09 & 799 \\
\hline $3(2 \mathrm{H})$-furanone, dihydro-2-methyl & 16.39 & 804 \\
\hline Methylpyrazine & 17.26 & 821 \\
\hline Furfural & 17.79 & 831 \\
\hline 2-Furanmethanol & 19.02 & 855 \\
\hline 2-Propanone, 1-(acetyloxy)- & 19.57 & 866 \\
\hline Furfurylformate & 21.49 & 903 \\
\hline 2.6-Dimethylpyrazine & 21.75 & 909 \\
\hline Ethylpyrazine & 21.99 & 914 \\
\hline 2.3-Dimethylpyrazine & 22.16 & 918 \\
\hline Propanoic acid, ethenyl ester & 24.05 & 958 \\
\hline 2-Furancarboxaldehyde, 5-methyl- & 24.24 & 963 \\
\hline 2-Furanmethanol, acetate & 25.60 & 992 \\
\hline Pyrazine, trimethyl- & 26.04 & 1001 \\
\hline Acetylpyrazine & 26.89 & 1021 \\
\hline 2-Ethyl-3,5-dimethylpyrazine ${ }^{\mathrm{b}}$ & 29.38 & 1080 \\
\hline 2-Methoxyphenol ${ }^{\mathrm{b}}$ & 29.89 & 1092 \\
\hline
\end{tabular}

a Major impact compound of coffee.

b Major impact compounds of coffee but \% area $<0.1$.

c Retention time (RT) and Kovats index (KI) for the CP-Sil 8 capillary column. 
air-tight and elastic cover seals the system and holds the devices in place from the back side. Due to the sensor design and the small gas volume of the micro array, the devices will respond easily to step changes in gas concentration within 1 or $2 \mathrm{~s}$. A photograph of the SAW micro array is shown in Fig. 1.

SPME/SAW: $2 \mathrm{~g}$ of coffee powder were filled in a $20 \mathrm{ml} \mathrm{HS}$-vial. After an incubation time of $15 \mathrm{~min}$ at $30^{\circ} \mathrm{C}$ in the incubator unit of an MPS2 autosampler (Gerstel) a 5 min during extraction in the headspace above the sample was performed with a $100 \mu \mathrm{m}$ polydimethylsiloxane (PDMS) SPME fibre (Supelco, Munich, Germany). Without cryogenic focusing the fibre was introduced into the injector system of the SAW-Sensor system. This injector system was heated to $220^{\circ} \mathrm{C}$. The volatiles were quickly desorbed and transported by a nitrogen gas flow to the micro array as described by Bender et al. (2003).

\section{Results and discussion}

In this section analytic data obtained from the coffee samples in different ways is presented: first using a GC/MS instrument which gave information about the single components of the VOCs composition. The degradation of the coffee samples was then successively monitored using a GC/FID instrument. By means of the panellists the information of the human response to the ageing samples was obtained and last but not least, the data obtained with a SAW sensor system was evaluated via a PCA algorithm.

A

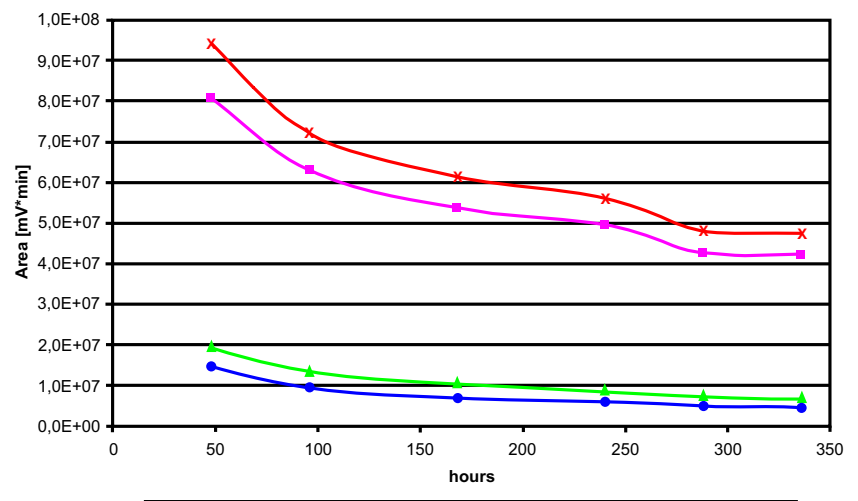

$\rightarrow-30^{\circ} \mathrm{C}$ identified $\quad=-60^{\circ} \mathrm{C}$ identified $\quad \longleftarrow-30^{\circ} \mathrm{C}$ total $\quad * 60^{\circ} \mathrm{C}$ total

C

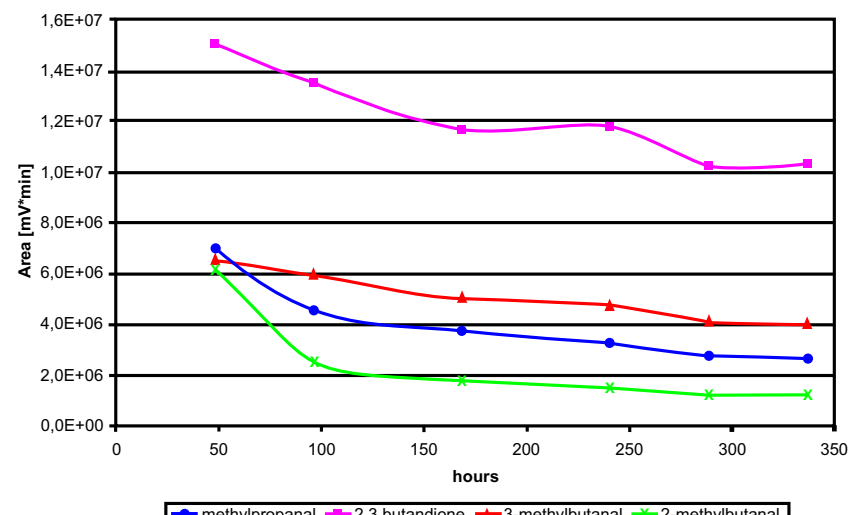

\subsection{Gas chromatography}

GC/MS analysis: in Table 1 only volatile compounds with a proportion greater than $0.1 \%$ of the total peak areas are listed, because these 30 volatiles may have an affinity towards the polymers of the sensors. Components with a smaller peak area will not influence the sensor signals, since their concentrations are in the lower ppb range, below the minimum detection limit of the SPME/SAW device. Only six of these 30 components (2,3-butandione, 2,3-pentandione, 3-methylbutanal, 2-methylbutanal, 2-ethyl-3,5-dimethylpyrazine and 2-methoxyphenol) are known as major impact compounds of coffee flavour (Blank, Sen, \& Grosch, 1992). For this reason also a semi-selective sensor approach, like SAW sensors, will not reflect coffee quality in its raw data.

GC/FID analysis: the change in headspace (HS) composition was clearly visible qualitatively and quantitatively during the storage of ground coffee, with investigated storage times between 2 and 14 days. Fig. 2 is showing in four different diagrams the effects of storage to this headspace composition. Both evaluation strategies at different sampling temperatures, determination of the overall headspace concentration (Fig. 2A) as well as the focussing on single components (Fig. 2B-D), showed a non-linear concentration decrease, which disables prediction of the coffee age. This behaviour was observed for both incubation temperatures $\left(30^{\circ} \mathrm{C}\right.$ and $60^{\circ} \mathrm{C}$ ) prior to the injection into the GC system. The chromatograms only showed differences in the peak size according to the
B

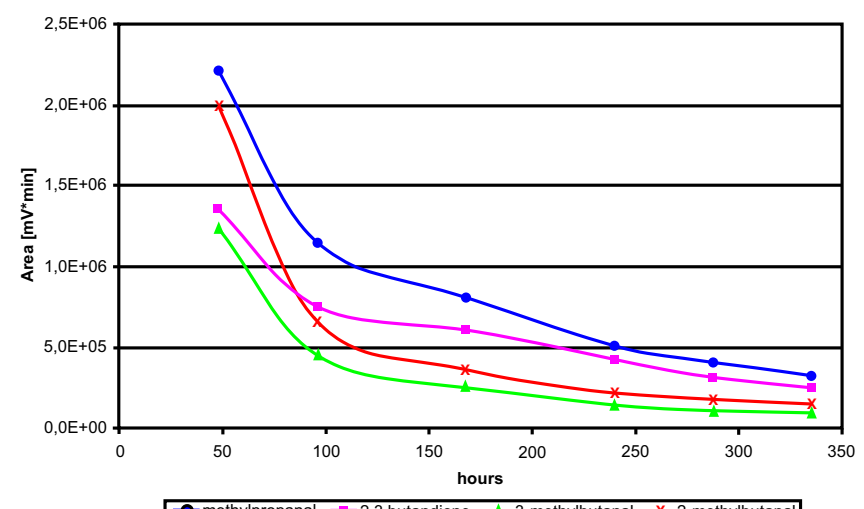

-0 -methylpropanal $-\frac{\mathrm{m}}{-2,3}$ butandione $\_$-methylbutanal $* 2$-methylbutanal

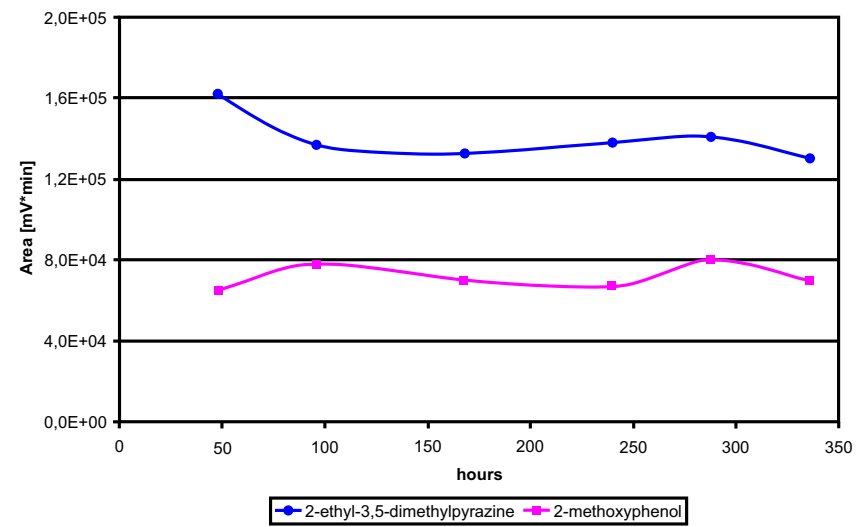

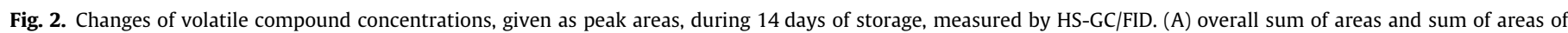

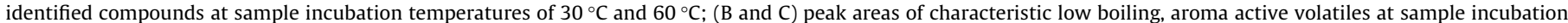
temperatures of $30^{\circ} \mathrm{C}$ and $60^{\circ} \mathrm{C}$ resp.; (D) peak areas of characteristic high boiling, aroma active volatiles at a sample incubation temperature of $60{ }^{\circ} \mathrm{C}$. 
different temperatures - more volatiles were present at higher temperatures.

In general, it should be taken into account that this time- and cost-intensive method requests a high operational knowledge.

\subsection{Sensory experiments}

Reference analyses were accompanied by sensory experiments conducted by six trained panellists. The triangular test revealed that the differences in coffee ageing were obvious, if the samples have larger time differences to each other. However, in contrast to the technical devices, the panellists were not able to differentiate between samples with a "ageing difference" smaller than $48 \mathrm{~h}$, except for each starting point of each ageing test: freshly grounded coffee samples compared to two days old coffee samples, respectively.

\subsection{Sensor system}

The implementation of a pre-concentration step is very advisable for food application, even if headspace sampling is used. Main reasons for using this method are low volatile concentrations from solid powders as aroma sources or the need of adjusting or controlling different humidity levels from the latter by the use of hydrophobic pre-concentration materials. For headspace sampling a pre-concentration with a proper material coated on a SPME fibre is the ideal combination. This approach was already successfully tested for other applications using SAW sensor arrays (Barié, Bücking, \& Rapp, 2006; Garcia-Gonzales, Barié, Rapp, \& Aparicio, 2006; Garcia-Gonzalez, Barié, Rapp, \& Aparicio, 2004).

The SPME method is based on the affinity of different headspace compounds into the coating of the fibre, i.e. a pre-selection step is available. Working with SPME-fibres showed a high reproducibility of corresponding measurements. Disadvantage of this technique is the high operational knowledge required to properly operate such a system as well as the higher time consumption of analysis. Although the SPME method was now included into the proposed sensor array based analytical set-up, the analytical time frame for each sample was still much shorter than with a GC/FID or GC/ MS setup. The reason for this is that the sensor array itself is able to differentiate between each sample provoked by the headspace concentration of the resulting mixture of volatiles. Thus, there is no waiting for long retention times of some single components like in the case of a GC/FID setup. Furthermore, a linear dependency between the total sensor signals and the age of the roasted ground

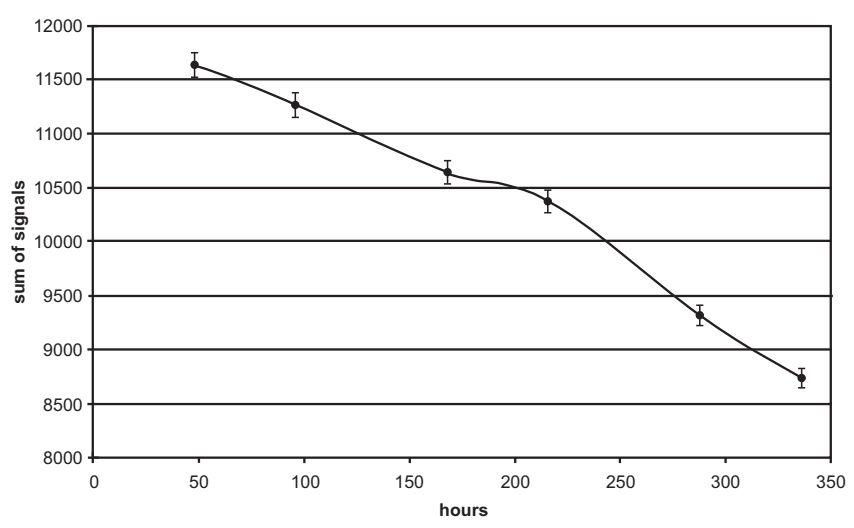

Fig. 3. Sum of selected SAW sensor signals for household blend coffee powder in different stages of storage (between 2 and 14 days). HS-vials contained $2 \mathrm{~g}$ of powder; incubation temperature was $30^{\circ} \mathrm{C}$ for $15 \mathrm{~min}$, SPME extraction $5 \mathrm{~min}$. coffee sample was clearly visible (Fig. 3). While flame ionisation detection is based upon parts per volume, the SAW sensor detection is based upon mass per volume. In this experimental sensor set-up the concentration of components with higher molecular weight remain nearly constant (Fig. 2D). As a consequence, compounds with a higher molecular weight influence strongly on the signal and a linear dependency is reached. For this reason coffee ageing could be observed with this analytical set-up much more effective than with the gas chromatographic set-up.

Moreover, PCA data evaluation on the basis of "In" values, depicted in Fig. 4, shows differences between three coffees (espresso, household blend, dark roasted arabica). Again the measurements were made of coffee samples with a storage time between 2 and 14 days - in total at eight different times of storage. Although a full separation by PCA was not achieved, differences are visible even in this two dimensional plot! Changes by time can be followed for each different coffee analysed. In addition a separation between dark roasted coffee/espresso coffee and the household blend coffee is obvious. Since espresso is also dark roasted coffee while the German average coffee is mild roasted, the differences are due to volatiles which increase during the last phase of the roasting process, when a mid-roasted coffee turns into a dark roasted coffee. These volatiles belong to the higher molecular fraction of the coffee aroma. Examples of chemical groups are pyrazines, phenolic compounds as well as furanones.

Experiments already show that the short term reproducibility is delivering very good results (i.e. 6-13 measurements in a row). Most of the industrial applications require a much longer time frame. Therefore, it is important to control in long-run experiments the behaviour of the sensors and add methods to calibrate the system, if necessary. However, we are aware of the fact that more research should be done in order to really proof the usefulness of our new approach. In the field of SAW and SPR driven biosensors an analytical validation and comparison of LOD/LOQ was done already with success (Lee, Kim, \& Kim, 2013).

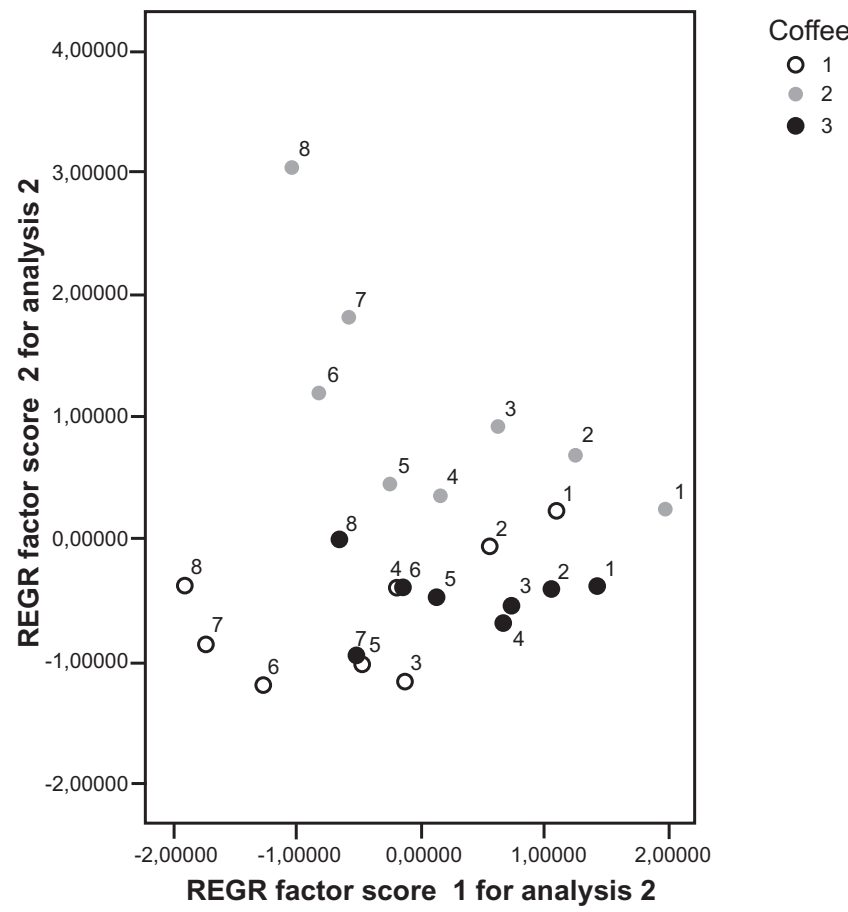

Fig. 4. PCA data evaluation on the basis of the ln values, coffee 1: espresso; coffee 2 : household blend; coffee 3: dark roasted arabica (Ethiopia). 


\section{Conclusion and outlook}

In this study, coffee ageing was observed by two different analytical techniques, gas chromatography and chemical sensors. In a third stage the obtained results with the technical systems were then compared and referred with a human sensory panel. Bottom line is that the new sensor based approach combining SPME enhanced sampling with a SAW sensor array based detector unit was capable to deliver directly a linear relation between volatile concentration and time of storage. Exactly this type of data is the most important for an end user who finds no low cost instrument available on the market, yet. So far these results are very promising, since the sensor based setup is rather simple, small and low cost compared to the examined conventional method using HSGC/FID or GC/MS. The latter of course would deliver a linear relationship with the selected data sets in combination with a proper statistical method. Therefore, the conventional methods are still a good reference tool for the ongoing research to establish sensor driven detectors with an enhanced focus towards an explicit characterisation of sensing polymers and their volatile compound affinities. Such work will optimise the composition of chosen sensitive polymer coatings with a second step in mind to develop a new analytical instrument for real industrial applications in food industry. Since the end-user in industry will be interested in an easy to operate hand-held unit we should also concentrate onto a user friendly and compact pre-concentration unit in replacement of the standard SPME approach out of headspace vials.

\section{Acknowledgements}

We acknowledge support by Deutsche Forschungsgemeinschaft and Open Access Publishing Fund of Karlsruhe Institute of Technology.

\section{References}

Avramov, I. D., Rapp, M., Voigt, A., Stahl, U., Dirschka, M. (2000). Comparative studies on polymer coated SAW and STW resonators for chemical gas sensor applications. In IEEE-EIA Int. Freq. Control Symp., Missouri, USA: Kansas City.

Baer, R. L., Flory, C. A. Tom-Moy, M., \& Solomon, D. S. (1992). STW chemical sensors. Proceedings of IEEE Ultrasonics Symposium, 293-298.

Barié, N., Bücking, M., \& Rapp, M. (2006). A novel electronic nose based on miniaturized SAW sensor arrays coupled with SPME enhanced headspaceanalysis and its use for rapid determination of volatile organic compounds in food quality monitoring. Sensors and Actuators B, 114, 482-488.

Barié, N., Wessa, T., Bruns, M., \& Rapp, M. (2004). Love waves in $\mathrm{SiO}_{2}$ layers on STW resonators based on $\mathrm{LiTaO}_{3}$. Talanta, 62, 71-79.

Bender, F., Barié, N., Romoudis, G., Voigt, A., \& Rapp, M. (2003). Development of a preconcentration unit for a SAW sensor microarray and its use for indoor air quality monitoring. Sensors and Actuators B, 93, 135-141.

Bender, F., Länge, K., Voigt, A., \& Rapp, M. (2004). Improvement of surface acoustic wave gas and biosensor response characteristics using a capacitive coupling technique. Analytical Chemistry, 76(13), 3837-3840.

Bicchi, C., Panero, O. M., Pellegrino, G. M., \& Vanni, A. C. (1997) Characterization of roasted coffee and coffee beverages by solid-phase microextraction - gas chromatography and principal component analysis. Journal of Agriculture and Food Chemistry, 45, 4680-4686.

Blank, I., Sen, A., \& Grosch, W. (1992). Potent odorants of the roasted powder and brew of arabica coffee. Zeitschrift für Lebensmittel-Untersuchung und -Forschung, 195, 239-245.

Bücking, M., Haugen, J.-E., \& Steinhart, H. (2003). Classification of peanuts by wet chemical analysis, instrumental methods, electronic nose devices and sensory analysis. In J. L. Le Quéré \& P. X. Etiévant (Eds.), Flavour research at the dawn of the twenty-first century, proceedings of the 10th Weurman flavour research symposium (pp. 528-531). Paris: Lavoisier, Editions Tec \& Doc.

Bücking, M., \& Steinhart, H. (2002). Characterization of the influence of different milk additives on the flavor release of coffee beverages by Headspace-GC and sensory analysis. Journal of Agriculture and Food Chemistry, 50, 1529-1534.

Costa Freitas, A. M., Parreira, C., \& Vilas-Boas, L. (2001). The use of an electronic aroma-sensing device to assess coffee differentiation - comparison with SPME gas chromatography-mass spectrometry aroma patterns. Journal of Food Composition and Analysis, 14, 513-522.

Deutsches Institut für Normung e.V (1995). DIN ISO 4120, Sensory analysis methodology - triangular test. Berlin, Germany: Beuth Verlag GmbH.
Garcia-Gonzales, D. L., Barié, N., Rapp, M., \& Aparicio, R. (2006). A fuzzy filter to study the selectivity and sensitivity of a SPME enhanced SAW sensor system characterizing virgin olive oil aroma. Sensors and Actuators B, 116, 49-54.

Garcia-Gonzalez, D. L., Barié, N., Rapp, M., \& Aparicio, R. (2004). Analysis of virgin olive oil volatiles by a novel electronic nose based on a miniaturized SAW Sensor array coupled with SPME enhanced headspace enrichment. Journal of Agricultural and Food Chemistry, 52(25), 7475-7479.

Grate, Jay W., Martin, Stephen J., \& White, M. Richard (1993a). Acoustic wave microsensors. Analytical Chemistry, 65(21), 940A-948A.

Grate, Jay W., Martin, Stephen J., \& White, M. Richard (1993b). Acoustic wave microsensors part II. Analytical Chemistry, 65(22), 987A-996A.

Gretsch, C., Delarue, J., Toury, A., Visani, P., Liardon, R. (1997). Detection of aroma above a coffee powder: Limits and perspectives of electronic sensors. In Proceedings of the 17 International Scientific Colloquium on Coffee, Paris (pp. 183-190).

Korhonová, M., Hron, K., Klimcíková, D., Müller, L., Bednár P., Barták P. (2009). Coffee aroma-statistical analysis of compositional data. Talanta, 80(2), 710-705. doi: 10.1016/j.talanta.2009.07.054. Epub 2009 Aug 3rd.

Kovacs, G., Vellekoop, M. J., Haueis, R., Lubking, G. W., \& Venema, A. (1994). A Love wave sensor for (bio)chemical sensing in liquids. Sensors and Actuators A, 43, $38-43$.

Lee, S., Kim, Y. I., \& Kim, K. B. (2013). Comparative study of binding constants from Love wave surface acoustic wave and surface plasmon resonance biosensors using kinetic analysis. Journal of Nanoscience and Nanotechnology, 13(11), 7319-7324 (ISSN: 1533-4880).

López-Galilea, I., Fournier, N., Cid, C., \& Guichard, E. (2006). Changes in headspace volatile concentrations of coffee brews caused by the roasting process and the brewing procedure. Journal of Agriculture and Food Chemistry, 54(22), $8560-8566$.

Martin, S. J., Ricco, A. J., Niemczyk, T. M., \& Frye, G. C. (1989). Characterization of SH acoustic plate mode liquid sensors. Sensors and Actuators, 20, 253-268.

Mondello, L., Costa, R., Tranchida, P. Q., Dugo, P., Lo Presti, M., Festa, S., et al. (2005). Reliable characterization of coffee bean aroma profiles by automated headspace solid phase microextraction-gas chromatography-mass spectrometry with the support of a dual-filter mass spectra library. Journal of Separation Science, 28(910), 1101-1109.

Nakanishi, K., Muguruma, H., \& Karube, I. (1996). A novel method of immobilizing antibodies on a quartz crystal microbalance using plasma polymerized films for immunosensors. Analytical Chemistry, 68, 1695-1700.

Pardo, M., Niederjaufner, G., Benussi, G., Comini, E., Faglia, G., Sberveglieri, G., et al. (2000). Data preprocessing enhances the classification of different brands of Espresso coffee with an electronic nose. Sensors and Actuators B, 69, 397-403.

Pawliszyn, J. (1997). Solid-phase microextraction theory and practice. New York, USA: Wiley.

Pawliszyn, J. (Ed.). (1999). Applications of solid-phase microextraction. London: Royal Society of Chemistry Monographs.

Pelusio, F., Nilsson, T., Montanarella, L., Tilio, R., Larsen, B., Facchetti, S., et al. (1995). Headspace solid-phase microextraction analysis of volatile organic sulfur compounds in black and white truffle aroma. Journal of Agriculture and Food Chemistry, 43, 2138-2143.

Rapp, M., Reibel, J., Voigt, A., Balzer, M., \& Bülow, O. (2000). New miniaturized SAWsensor array for organic gas detection driven by multiplexed oscillators. Sensors and Actuators B, 65, 169-172.

Semmelroch, P., \& Grosch, W. (1996). Studies on character impact odorants of coffee brews. Journal of Agriculture and Food Chemistry, 44, 537-543.

Snow, N. H., \& Slack, G. C. (2002). Headspace-analysis in modern gas chromatography. Trends in Analytical Chemistry, 21, 608-617.

Wessa, T., Küppers, S., Rapp, M., \& Reibel, J. (2000). Validation of an industrial analytical sensor procedure realized with a SAW based sensor system. Sensors and Actuators B, 70, 203-213 (special issue in memoriam of Prof. Göpel).

Wohltjen, Hank (1984). Mechanism of operation and design considerations for surface acoustic wave device vapour sensors. Sensors and Actuators, 5(4), 307-325.

Wohltjen, H., \& Dessy, R. E. (1979). Surface acoustic wave probe for chemical analysis. Analytical Chemistry, 51, 1458-1475.

Yang, X., \& Peppard, T. (1995). Solid-phase microextraction of flavor compounds-a comparison of two fiber coatings and a discussion of the rules of thumb for adsorption. $L C / G C, 13,882-886$.

Nicole Barié studied chemistry at the University of Karlsruhe, Germany. In 1995 she received her diploma. She worked at the Forschungszentrum Karlsruhe in the field of biosensors as a Ph.D. student. In 1998 she obtained her Ph.D. As a postdoc, she worked in the application development for gas- and biosensors. Her fields of interest include immunosensing with SAW devices and the development of a preconcentration unit for gas sensor arrays. Since 2008 she is working as a project manager at the Institute of Microstructure Technology (IMT) of Karlsruhe Institute of Technology (KIT) in the area of micro- and nanostructured mold inserts.

Mark Bücking studied food chemistry at the University of Hamburg, Germany, where he received his 2nd State Examination in 1997 and his Ph.D. in 1999. From 2000 to 2001 he worked as a research scientist at the Institut für Qualitätssicherung $\mathrm{GmbH}$, Hamburg, Germany. Until 2004 he was a flavour research scientist and project leader at Agrotechnology and Food Innovations B.V., Wageningen, The Netherlands. Since 2004 he is Head of the Department for Environmental and Food Analyzes at Fraunhofer IME Schmallenberg, Germany. His fields of interest include industrial food safety, fast detection methods and chromatographic trace analysis. 
Ullrich Stahl studied chemistry at the Technical University of Karlsruhe, Germany He received the Ph.D. degree in 1999. During his Ph.D. studies at the Institute for Instrumental Analysis of the Forschungszentrum Karlsruhe, Germany, he focused on methods to enhance the stability of polymer films on SAW-sensors as well as new coating techniques. He worked as a member of the R\&D group SAW based electronic noses. Afterwards he joined the R\&D group of analytical metal oxide micro gas sensors at the Forschungszentrum Karlsruhe. Presently he is working as a Professor in the fields of organic and instrumental analytical Chemistry at the Central University of Ecuador.
Michael Rapp studied physics in Stuttgart and Heidelberg, Germany, where he obtained his Diploma in 1987. During his thesis at the University of Heidelberg and BASF company, Ludwigshafen, he developed SAW sensors mainly for inorganic gases such as sensors for $\mathrm{NO}_{2}$ detection. In 1991, he obtained his Ph.D. degree and became a Scientific Manager at Forschungszentrum Karlsruhe, Germany, where he established a SAW sensor group developing several analytical SAW sensor systems for gas and liquid phase. His research programme is very close to the practical needs and commercialization of such sensor systems. 